\section{Performance assessment of health examination for freshmen}

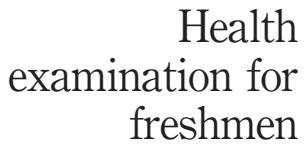

K.S. Chen

Department of Industrial Engineering and Management, National Chin-Yi Institute of Technology, Taichung, Taiwan, ROC

H.T. Chen

Department of Information Management, Chang Jung University, Tainan, Taiwan, $R O C$

L.L. Wu

National Chin-Yi Institute of Technology, Taichung, Taiwan, ROC, and

J.T. Emanuel

Department of Industrial and Manufacturing Engineering and Technology, Bradley University, Peoria, Illinois, USA

\begin{abstract}
Purpose - A health examination is one of important events for freshmen when they enter a university. Consequently, the quality and time required for the examination are components in any index measuring the service performance of a university. However, performance measure for the health examination with a performance index is comparatively seldom. This paper aims to provide an index to measure health examination performance for freshmen.

Design/methodology/approach - A health examination performance index (HEPI) is defined for a university's health examination procedure. To make accurate use of the HEPI in assessing health examination performance, this paper constructs a uniformly minimum variance unbiased (UMVU) estimator of the HEPI using an exponential distribution to develop a hypothesis testing procedure.

Findings - Since health examination time exhibits the smaller-the-better type quality characteristic of time orientation, the HEPI is appropriate for evaluating the hospital's performance in providing freshmen health examinations. This study also investigates the relationship between the HEPI and the non-conformance rate of health examination performance, and tabulates the non-conformance rate under the exponential distribution.

Originality/value - The proposed testing procedure is easily applied and can accurately evaluate whether the true health examination performance for a hospital meets a university's requirement. It can also be used to select a qualified outsourcing hospital and improve the service provided by the school.
\end{abstract}

Keywords Exponential distribution, Performance measures, Personal health, Students

Paper type Technical

\section{Introduction}

Due to the increased importance placed on the global market, customer service quality has become increasingly important. Providing service quality that satisfies the

The authors would like to thank the National Science Council of the Republic of China for financially supporting this research under contract No. NSC-89-2416-H-167-006.
International Journal of Quality \& Reliability Management Vol. 22 No. 8,2005 pp. $849-859$ (C) Emerald Group Publishing Limited 0265-671X DOI 10.1108/02656710510617256 
$\mathrm{IJQRM}$

22,8

\section{0}

customer is the challenge for today's service industry. To improve customers' satisfaction, numerous scholars have devoted themselves to researching the properties of the service industry in order to increase service quality and performance. Juran (1988) proposed a definition of service as "work performed for someone else." Ishikawa (1990) proposed "services are efficient works that do not produce physical products." Kotler (1988) stated:

A service is any act or performance that one party can offer to another that is essentially intangible and does not result in the ownership of anything. Its production may or may not be tied to a physical product.

In addition, LaLonde and Zinszer (1976) pointed out that customer service could be viewed as an activity as an indication of performance levels and as a philosophy of management. LaLonde et al. (1988) defined customer service as:

... a process for providing significant value-added benefits to the supply chain in a cost-effective way.

As noted by Dickson (1966) and Weber et al. (1991), product quality and delivery speed are two major concerns used to evaluate suppliers' performance. Weber's (1991) survey pointed out that quality and delivery time is the two most important factors selecting a supplier. Hence, customers expect high quality service and short service time from today's service industries. Superior service quality is the key to being able to compete with others in the market. Short service time can both enhance the competitive strength and win market share for an advanced service process. All customers dislike waiting in line at the supermarket checkout, at the local bank, or at a fast food restaurant. Adding more checkout clerks, bank tellers, or servers is not always the most economical strategy for improving service performance; however, managers need to determine ways to keep service times within acceptable limits.

Teaching, research and service are three major functions for a university. In addition to nurturing teaching and research quality, improving the service provided by the administrative departments can make the school more attractive to potential students. The teaching and administrative staff and students are the main service customers of an institution's administrative department. The service provided to students includes academic counseling and registration, academic record keeping, personal and career counseling and health services. To prevent the spread of diseases and to provide a healthy environment, the health examination is one of the first required activities for freshmen that enter a university. The time required for the health examination and the quality of such an examination are important in assessing the service performance of a university. A university often has to select a hospital to provide health examination services before freshmen register. In the selection and certification of the hospital, one key issue is the capability of the hospital to provide timely and high quality health examinations. Process capability analysis is a convenient and powerful tool for measuring the process capability and performance. Hence, process capability indices (PCIs) have been developed and used in numerous industries. Many quality engineers and statisticians, such as Kane (1986), Chan et al. (1988), Boyles (1991), Pearn et al. (1992), Vännman (1995), Helmut et al. (1996), Pearn and Chen (2002) have proposed methodologies to assess product/process capability.

In general, the tasks involved in the health examination for a freshman can be divided into the 11 components shown in Table I. 


\begin{tabular}{|c|c|c|}
\hline Item & Health examination tasks & exami \\
\hline 1 & Filling out form & freshme \\
\hline 2 & Measuring height and weight & \\
\hline 3 & Testing hearing acuity and color blindness & \\
\hline 4 & Testing blood pressure & \\
\hline 5 & Testing visual acuity & 85 \\
\hline 6 & Recording dental history & \\
\hline 7 & Recording family medical history & \\
\hline 8 & Drawing blood & \\
\hline 9 & Testing urine & Table \\
\hline 10 & Examining X-ray & Examination tasks ar \\
\hline 11 & Completing and returning the form & sequence \\
\hline
\end{tabular}

The processing time for each health examination item directly influences total health examination time for a freshman. Health examination time is defined as the total time from beginning item 1 to the completion of item 11 . The shorter the processing time of each item implies that the shorter total health examination time and the better the service performance. In this study, a health examination performance index (HEPI, i.e. PCI) is initially defined, and the UMVU estimator of HEPI is derived under the assumption that the service times follow an exponential distribution. This estimator is then used to construct the one-to-one relationship between the HEPI and the non-conformance rate of health examination performance. Finally, an efficient hypothesis testing procedure of HEPI is established. The hypothesis testing procedure allows a hospital to assess whether health examination performance satisfies the customers' requirement. The HEPI can also be used to help a university to select qualified hospitals. A corresponding table of the non-conformance rate of health examination performance is also provided to verify that the hospitals meet the required HEPI.

The rest of this paper is organized as follows. The following section defines and introduces the HEPI under the assumption that service time follows an exponential distribution, and then discusses the relationship between the HEPI and the non-conformance rate of health examination performance. The third section derives the estimator of the HEPI. The fourth section constructs a hypothesis testing procedure for the HEPI. The final section provides a conclusion.

\section{The relationship between the HEPI and the non-conformance rate of health examination performance}

Generally, health examination time is not constant. Assume that health examination time $(X)$ for a freshman is a random variable. The unit time is expressed in minutes. To improve health examination performance, the time for each examination item is required to be less than $U_{i}$ minutes in order to keep $\sum_{i=1}^{k} U_{i} \leq U$, where $U$ is the upper limit for total health examination time. If service quality remains constant, then the shorter examination time represents the better health examination performance. Hence, health examination time is a smaller-the-better type quality characteristic. Montgomery (1991) developed a capability index $S_{I}$ for measuring the smaller-the-better type quality characteristic 
$\mathrm{IJQRM}$

22,8

852 that can be used to evaluate whether health examination performance meets the requirement. $S_{I}$ is defined as follows:

$$
S_{I}=\frac{U-\mu}{\sigma}
$$

where $\mu$ denotes the average health examination time, $\sigma$ represents the standard deviation of health examination time, and $U$ is the upper limit of health examination time. Karlin and McGregor (1958) and Hiller and Lieberman (1986) state that service time generally follows an exponential distribution. Since the mean $\mu$ and standard deviation $\sigma$ are both $\lambda$ in the exponential distribution, then the health examination performance index (HEPI) $S_{I}$ can be written as:

$$
S_{I}=\frac{U-\mu}{\sigma}=\frac{U-\mu}{\lambda}=\frac{U}{\lambda}-1 .
$$

When the mean examination time $\lambda \leq U$, then $S_{I} \geq 0$. Conversely, when $\lambda>U$, then $S_{I}<0$. Obviously, the smaller the $\lambda$, the smaller the $\mu$ and $\sigma$ is, and the larger $S_{I}$ is. The larger the $S_{I}$ implies that the better the health examination performance.

If health examination time for a freshman, $X$, exceeds the upper limit of health examination time (i.e. $X>U$ ), then the health examination performance is out of control. The probability of being out of control is defined as the non-conformance rate for health examination performance. When the non-conformance rate of health examination performance is high, freshmen will experience some long waiting lines and service quality and performance for this university will decline. This may result in the outsourcing hospital not being selected by this university next year. The non-conformance rate of health examination performance, $p$, is the $\operatorname{Pr}(X>U)$. The relationship between the HEPI $S_{I}$ and the non-conformance rate of health examination performance $p$ under the assumption of exponential distribution can be represented as follows:

$$
p=P(X>U)=\exp \left\{-\left(S_{I}+1\right)\right\},-1<S I<\infty .
$$

Clearly, a one-to-one mathematical relationship exists between the index $S_{I}$ and the non-conformance rate, $p$. Figure 1 represents a functional graph of equation (3), and shows that $p$ is a strictly decreasing function of $S_{I}$. The larger the index value $S_{I}$, the smaller the non-conformance rate of health examination performance $p$ and the smaller the probability that freshmen health exams require a long wait.

Table II lists various $S_{I}$ values and the corresponding non-conformance rates $p$ for health examination performance. For the $S_{I}$ values that are not listed in Table II, the $p$ values can be obtained through interpolation. The $p$-value for a university/hospital can be calculated by dividing the non-conformance number of health examination by the total number of sampled freshmen. A smaller $p$ requires a larger sample size in order to estimate precisely its value (see, Montgomery, 1991, for details). Therefore, utilizing the one-to-one relationship between $S_{I}$ and $p$, the $S_{I}$ index can be a convenient and effective tool not only for estimating the non-conformance rate $p$, but also for evaluating health examination performance of a university/hospital. 


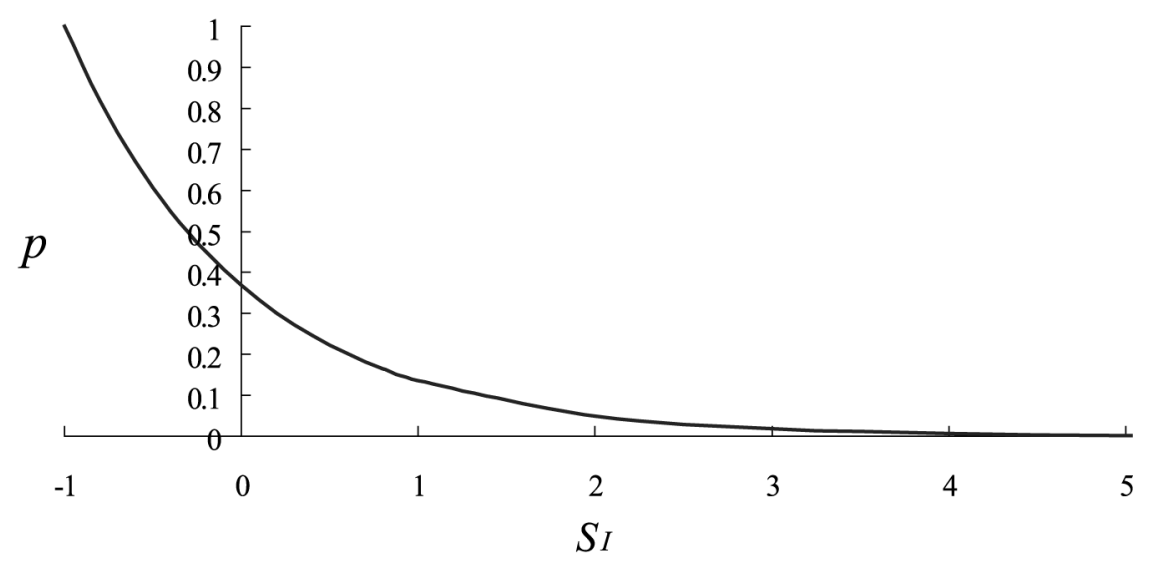

\section{Health examination for freshmen}

853

Figure 1.

The functional relationship graph between $p$ and $S_{I}$

\begin{tabular}{lccc}
\hline HEPI $\left(S_{I}\right)$ & Non-conformance rate $(p)$ & HEPI $\left(S_{I}\right)$ & Non-conformance rate $(p)$ \\
\hline$\infty$ & 0.000 & 0.25 & 0.287 \\
3.00 & 0.018 & 0.00 & 0.368 \\
2.75 & 0.024 & -0.10 & 0.407 \\
2.50 & 0.030 & -0.20 & 0.449 \\
2.25 & 0.039 & -0.30 & 0.497 \\
2.00 & 0.050 & -0.40 & 0.549 \\
1.75 & 0.064 & -0.50 & 0.607 \\
1.50 & 0.082 & -0.70 & 0.670 \\
1.25 & 0.105 & -0.80 & 0.741 \\
1.00 & 0.135 & -1.00 & 0.819 \\
0.75 & 0.174 & 0.905 & Table II. \\
0.50 & 0.223 & & $S_{I}$ vs $p$ \\
\hline
\end{tabular}

Hence, the HEPI $\left(S_{I}\right)$ value for a university not only can be a measure of performance for administering health examinations, but can also be used to select a hospital to administer the examinations. For example, if a university requires a non-conformance rate $(p)$ for health examinations is less than 5 percent, then referring to Table II shows an equivalent HEPI of 2.00. If the HEPI exceeds 2.00, then the hospital will be a candidate for performing freshman health examinations.

\section{Estimation of the HEPI}

Because the population mean and standard deviation of health examination time for freshmen are generally unknown, they must be estimated in practice. Let $X_{j}$ represents health examination time of the $j$ th freshman, then $\left(X_{1}, X_{2}, \ldots, X_{n}\right)$ is a random sample taken from the exponential distribution with mean of $\lambda$ units of time. If the sample mean $\bar{X}$ is used to estimate the population mean, $\lambda$, then the estimated index of $S_{I}$ can be written as:

$$
\hat{S}_{I}=\frac{U}{\bar{X}}-1, \text { where } \bar{X}=\left(\sum_{j=1}^{n} X j\right) / n \text {. }
$$


IJQRM

22,8

854

The characteristic function of the exponential distribution is $\phi(t)=E\left(e^{i t X_{j}}\right)=(1-i \lambda t)^{-1}$, therefore the characteristic function of the distribution of $\bar{X}$ can be derived as follows:

$$
\begin{aligned}
\phi_{\bar{X}}(t) & =E\left(e^{i t \bar{X}}\right)=E\left(e^{i\left(\frac{t}{n}\right) \sum_{j=1}^{n} X_{j}}\right)=E\left(\prod_{j=1}^{n} e^{i\left(\frac{t}{n}\right) X_{i}}\right)=\left[E\left(e^{i\left(\frac{t}{n}\right) X_{j}}\right)\right]^{n} \\
& =\left(1-i\left(\frac{\lambda}{n}\right) t\right)^{-n} .
\end{aligned}
$$

Clearly, $\phi_{\bar{X}}(t)$ is the characteristic function of Gamma $(n, \lambda / n)$, and hence $\bar{X}$ possesses a gamma distribution with parameters $n$ and $\lambda / n$. The expectation of $\hat{S}_{I}$ can then be derived as:

$$
E\left(\hat{S}_{1}\right)=E\left(\frac{U}{\bar{X}}-1\right)=U \times E\left(\bar{X}^{-1}\right)-1
$$

Because $E\left(\bar{X}^{-1}\right)=n / \lambda(n-1)$, the expectation of $\hat{S}_{I}$ can be expressed as:

$$
E\left(\hat{S}_{I}\right)=\left(\frac{n}{n-1}\right)\left(\frac{U}{\lambda}\right)-1
$$

Obviously, $\hat{S}_{I}$ is not an unbiased estimator of $S_{I}$, since $E\left(\hat{S}_{I}\right) \neq S_{I}$. But when $n$ becomes large, $E\left(\hat{S}_{I}\right)$ approximates $S_{I}$. Therefore, $\hat{S}_{I}$ is an approximate unbiased estimator of $S_{I}$ when $n$ becomes large. Then, $\hat{S}_{I}$ can be modified as follows:

$$
\tilde{S}_{I}=\left(\frac{n-1}{n}\right) \frac{U}{\bar{X}}-1
$$

Hence, $\tilde{S}_{I}$ is not only the unbiased estimator of $S_{I}$, but also a function of the complete and sufficient statistic $\bar{X}$. Thus, $\tilde{S}_{I}$ is the best estimator (i.e. minimum variance unbiased estimator (UMVU estimator)) of $S_{I}$ (see Appendix 1 for details). Let $Y=\widetilde{S}_{I}$, then the probability density function of $\tilde{S}_{I}$ can be derived as follows (see appendix 2 for details):

$$
f_{Y}(y)=\frac{\left[(n-1)\left(S_{I}+1\right)\right]^{n}}{\Gamma(n)(y+1)^{(n+1)}} \times \exp \left\{-\frac{(n-1)\left(S_{I}+1\right)}{y+1}\right\},-1<y<\infty .
$$

Meanwhile, the $r$ th moment of $\tilde{S}_{I}$ can be derived as follows (see appendix 3 for details):

$$
E\left(\tilde{S}_{I}\right)^{r}=\sum_{i=0}^{r} C_{i}^{r}\left\{(-1)^{r-i}\left[(n-1)\left(S_{I}+1\right)\right]^{i}(\Gamma(n-i) / \Gamma(n))\right\} .
$$


By the $r$ th moment of $\tilde{S}_{I}$, the variance of $\tilde{S}_{I}$ can be obtained as:

$$
\operatorname{Var}\left(\tilde{S}_{I}\right)=\left(\frac{\left(S_{I}+1\right)^{2}}{n-2}\right), n>2 .
$$

\section{Hypothesis testing procedure for the HEPI}

Many quality engineers and statisticians simply utilize the point estimates of PCIs calculated from the sample data and then make a conclusion whether a process capability meets the requirement. This approach is not reliable since sampling errors are ignored (Pearn and Chen, 2002). Hence, taking the sampling errors into account, Cheng (1992, 1995), Pearn and Chen (2002) have developed simple but practical procedures for $C_{p u}, C_{p l}$, and $C_{p k}$, to assist the practitioner in detecting whether their processes meet the capability requirement. Thus, a statistical testing procedure is needed to objectively assess whether health examination performance meets the required level. Assuming that the required HEPI value for a hospital is larger than or equal to $s$, where $s$ denotes the target value, then the hypothesis testing procedure for testing $H_{0}: S_{I} \leq s$ (health examination performance is not capable) vs $H_{1}: S_{I}>s$ (health examination performance is capable) can be developed. Suppose $U$ is known, using $\tilde{S}_{I}$ as the test statistic, and the sample mean $\bar{X}=\sum_{j=1}^{n} X / n$ can be calculated based on $n$ sample observations. Hence, the estimated value of $\tilde{S}_{I}, s_{0}$, can be obtained. Under the specified significance level $\alpha$ (the producer risk), the $p$-value of the test statistic, $\tilde{S}_{I}$, can be obtained as follows:

$$
\begin{aligned}
p-{ }_{I}>s_{0} \mid S_{I} & =s=\operatorname{Pr}\left\{\left(\frac{n-1}{n}\right) \cdot\left(\frac{U}{\bar{X}}\right)-1>s_{0} \mid S_{I}=s\right\} \\
& =\operatorname{Pr}\left\{\bar{X}<\frac{(n-1) U}{n\left(s_{0}+1\right)} \mid \lambda=\frac{U}{1+s}\right\} .
\end{aligned}
$$

Let $W=n \bar{X} / \lambda$, then $W$ possesses Gamma ( $n, 1)$ (see appendix 4 for details). Substituting $W=n \bar{X} / \lambda$ in equation (12), a statistical software package, SAS can be employed to calculate the $p$-value as follows:

$$
p-\text { value }=\operatorname{Pr}\left\{W<(n-1)(1+s) /\left(1+s_{0}\right)\right\}=\operatorname{PROBGAM}(k, n),
$$

where $k=(n-1)(1+s) /\left(1+s_{0}\right)$ and $\operatorname{PROBGAM}(k, n)$ is a cumulative probability which is lower cumulated by $k$ of gamma distribution with $n$ and 1 degree of freedom in $S A S$. As $k$ and $n$ are known, the studied $p$-value can easily be calculated.

To let universities/hospitals conveniently assess whether the health examination performance for freshmen meets the required target value (i.e. $H_{0}: S_{I} \leq s$ vs $H_{1}: S_{I}>s$ ), the proposed testing procedure can be organized as follows (Cheng, 1995):

- Step 1. Determine the upper limit of health examination time $U$, the HEPI value $s$ and the sample size $n$.

- Step 2. Specify a significance level $\alpha$.

- Step 3.Take a sample of size $n$ and calculate the sample mean $\bar{X}=\sum_{j=1}^{n} X / n$, then the value of test statistic, $\widetilde{S}_{I}$, which is denoted by $s_{0}$, can be obtained. examination for
freshmen 
$\mathrm{IJQRM}$

22,8

\section{6}

- Step 4. Obtain the $p$-value by using SAS, according to the value $s$, sample size $n$ and $s_{0}$ value (see appendix 5 for details).

- Step 5. Compare $p$-value with $\alpha$ value, and draw a conclusion. The decision rules are as follows:

- If $p$-value $\leq \alpha$, we conclude that the hospital's HEPI meets the required target value for a university (or health examination performance is qualified).

- If $p$-value $>\alpha$, we conclude that the hospital's HEPI does not meet the required target value for a university (or health examination performance is not qualified).

Based on the proposed testing procedure, health examination performance of freshmen is easy to assess. The following example illustrates the use of the testing procedure. To satisfy a university's serious concern regarding health examination performance and service quality, suppose the non-conformance rate of health examination performance $p$ for a hospital is required to be less than 8.2 percent. Referring to Table II, a $S_{I}$ value of 1.5 is obtained. Thus, in step 1, the HEPI value is set at $s=1.5$. Furthermore, assume that a sample of size $n=20$ is obtained and $U$ is known. By specifying the significance level $\alpha=0.01$ in step 2 , the value $s_{0}$ of test statistic $\tilde{S}_{I}$ can be calculated from the sample data in step 3. In step 4, $p$-value is obtained from $S A S$ with specified $n, s$ and $s_{0}$. Finally, step 5 compares $p$-value with 0.01 and draws a conclusion about the hypotheses. If $p$-value $\leq 0.01$, the university concludes that the true HEPI value meets the requirement or health examination performance is qualified for a hospital. Otherwise, health examination performance for a hospital is concluded to be not qualified. This testing procedure can be easily employed by a hospital to measure its own performance and it can also be useful for a university when selecting a hospital to perform freshman medical examinations.

\section{Conclusion}

Since health examination time exhibits the smaller-the-better type quality characteristic of time orientation (Montgomery, 1991), the capability index $S_{I}$ (i.e. HEPI) is appropriate for evaluating the hospital's performance in providing freshmen health examinations. To utilize the HEPI, $S_{I}$, in effectively and accurately assessing health examination time under an exponential distribution, some properties of the HEPI, $S_{I}$, are discussed. This study also investigates the relationship between the index $S_{I}$ and the non-conformance rate of health examination performance $p$, and tabulates the non-conformance rate of health examination performance under the exponential distribution. Furthermore, a uniformly minimum variance unbiased (UMVU) estimator of $S_{I}$ is derived in this study. The UMVU estimator of $S_{I}$ is then utilized to construct the hypothesis testing procedure. The proposed testing procedure is easily applied and can accurately evaluate whether the true health examination performance for a hospital meets a university's requirement. A university can use this procedure to select a qualified outsourcing hospital. This study also provides a table of the performance index $S_{I}$ with its corresponding non-conformance rate of health examination performance $p$. Hence, for any specified $p$, a corresponding $S_{I}$ value can be obtained, and the 
proposed testing procedure can be expressed in terms of the non-conformance rate of health examination performance. The testing procedure proposed in this paper can also be applied to other activities for a university or hospital.

\section{Health examination for freshmen}

\section{References}

Boyles, R.A. (1991), “The Taguchi capability index”, Journal of Quality Technology, Vol. 23, pp. 17-26.

Chan, L.K., Chang, S.W. and Spiring, F.A. (1988), "A new measure of process capability: $C_{p m}$ ", Journal of Quality Technology, Vol. 20 No. 3, pp. 162-75.

Cheng, S.W. (1992), "Is the process capable? Tables and graphs in assessing $C_{p m}$ ", Quality Engineering, Vol. 4 No. 4, pp. 563-76.

Cheng, S.W. (1995), "Practical implementation of the process capability indices", Quality Engineering, Vol. 7 No. 2, pp. 239-59.

Dickson, G.W. (1966), "An analysis of vendor selection systems and decisions”, Journal of Purchasing, Vol. 2 No. 1, pp. 5-17.

Helmut, S., James, P. and Cliff, L. (1996), "Uses of process capability indices in the supplier certification process”, Quality Engineering, Vol. 8 No. 2, pp. 225-35.

Hiller, F.S. and Lieberman, G.J. (1986), Introduction to Operations Research, McGraw-Hill, New York, NY.

Ishikawa, K. (1990), Introduction to Quality Control, Chapman \& Hall, London.

Juran, J.M. (1988), Juran's Quality Control Handbook, 4th ed., McGraw-Hill, New York, NY.

Kane, V.E. (1986), "Process capability indices”, Journal Quality Technology, Vol. 18, pp. 41-52.

Karlin, S. and McGregor, J. (1958), "Many server queuing processes with poisson input and exponential service time", Pacific Journal of Mathematics, Vol. 8, pp. 87-118.

Kotler, P. (1988), Marketing Management: Analysis, Planning, Implementation and Control, Prentice-Hall, Englewood Cliffs, NJ.

Lalonde, B.J. and Zinszer, P.H. (1976), Customer Service: Meaning and Measurement, National Council of Physical Distribution Management, Chicago, IL.

Lalonde, B.J., Cooper, M.C. and Noordewier, T.G. (1988), Customer Service: A Measurement Perspective, Council of Logistics Management, Chicago, IL.

Montgomery, D.C. (1991), Introduction to Statistical Quality Control, Wiley, New York, NY.

Pearn, W.L. and Chen, K.S. (2002), "One-sided capability indices $C_{p u}$ and $C_{p l}$. decision making with sample information”, International Journal of Quality \& Reliability Management, Vol. 19 No. 3, pp. 221-45.

Pearn, W.L., Kotz, S. and Johnson, N.L. (1992), "Distributional and inferential properties of process capability indices”, Journal of Quality Technology, Vol. 24, pp. 216-31.

Vännman, K. (1995), “A unified approach to capability indices”, Statist. Sinica, Vol. 5, pp. 805-20.

Weber, C.A., Current, J.R. and Benton, W.C. (1991), "Vendor selection criteria and methods", European Journal of Operational Research, Vol. 50, pp. 2-18.

\section{Further reading}

Bothe, D.R. (1999), “A capability index for multiple process streams”, Quality Engineering, Vol. 11 No. 4, pp. 613-8. 
IJQRM

22,8

858

\section{Appendix 1. $\tilde{S}_{I}$ is a UMVU estimator of $S_{I}$}

(1) $\tilde{S}_{I}$ is an unbiased estimator of $S_{I}$.

Since $\bar{X}$ possesses a gamma distribution with parameters $n$ and $\lambda / n, E\left(\bar{X}^{-1}\right)$ can be derived as:

$$
\begin{gathered}
E\left(\bar{X}^{-1}\right)=\int_{0}^{\infty} \bar{x}^{-1} \cdot \frac{1}{\Gamma(n)(\lambda / n)^{n}} \bar{x}^{(n-1)} e^{-n \bar{x} / \lambda} d x \\
=\frac{n}{\lambda(n-1)} \int_{0}^{\infty} \frac{1}{\Gamma(n-1)(\lambda / n)^{n-1}} \bar{x}^{(n-1)-1} e^{-n \bar{x} / \lambda} d x \\
=\frac{n}{\lambda(n-1)} .
\end{gathered}
$$

Thus, $E\left(\tilde{S}_{I}\right)=E\left[\left(\frac{n-1}{n}\right) \cdot \frac{U}{\bar{X}}-1\right]=\left(\frac{n-1}{n}\right) \cdot U \cdot E\left(\bar{X}^{-1}\right)-1=\frac{U}{\lambda}=S_{I}$, hence, $\tilde{S}_{I}$ is an unbiased estimator of $S_{I}$.

(2) $\bar{X}$ is a complete and sufficient statistic of $\lambda$.

Since $\bar{X} \sim \operatorname{Gamma}(n, \lambda / n)$, thus:

$$
\begin{aligned}
f(\bar{x} ; \lambda) & =\frac{1}{\Gamma(n)(\lambda / n)^{n}} \bar{x}^{(n-1)} e^{-n \bar{x} / \lambda} \\
& =\exp \{(n-1) \ln x-n \bar{x} / \lambda+[n \ln n-\ln \lambda-\ln \Gamma(n)]\},
\end{aligned}
$$

then, Gamma $(n, \lambda / n)$ is an exponential family. Consequently, $\bar{X}$ is a complete and sufficient statistic of $\lambda$. $\tilde{S}_{I}$ is a function of $\bar{X}$. According to (1) and (2), $\tilde{S}_{I}$ is a UMVU estimator of $S_{I}$.

\section{Appendix 2. The probability density function of $\tilde{\boldsymbol{S}}_{I}$}

$$
\text { Let } Y=\tilde{S}_{I}=\left(\frac{n-1}{n}\right) \cdot\left(\frac{U}{\bar{X}}\right)-1 \text {, then } \bar{X}=\frac{(n-1) U}{n(Y+1)} \text { and } \frac{d \bar{X}}{d Y}=\frac{-(n-1) U}{n(Y+1)^{2}} \text {. }
$$

Utilizing transformations of random variables, the probability density function of $Y$ can be obtained as follows:

$$
\begin{aligned}
f_{Y}(y) & =f_{\bar{X}}(\bar{x})\left|\frac{d \bar{x}}{d y}\right|=f_{\bar{X}}\left(\frac{(n-1) U}{n(y+1)}\right)\left|\frac{-(n-1) U}{n(y+1)^{2}}\right| \\
& =\frac{1}{\Gamma(n)(\lambda / n)^{n}}\left(\frac{(n-1) U}{n(y+1)}\right)^{n-1} e\left(\frac{-(n-1) U}{(y+1) \lambda}\right) \cdot \frac{(n-1) U}{n(y+1)^{2}} \\
& =\frac{\left[(n-1)\left(S_{I}+1\right)\right]^{n}}{\Gamma(n)(y+1)^{n+1}} \exp \left[-\frac{(n-1)\left(S_{I}+1\right)}{y+1}\right],-1<y<\infty .
\end{aligned}
$$

\section{Appendix 3. The $\boldsymbol{r}$ th moment of $\tilde{S}_{I}$}

The $r$ th moment of $\tilde{S}_{I}$ is derived as follows:

$$
E\left(\tilde{S}_{I}\right)^{r}=E\left(\left(\frac{n-1}{n}\right) \cdot\left(\frac{U}{\bar{X}}\right)-1\right)^{r}=\sum_{i=0}^{r} C_{i}^{r}\left\{(-1)^{r-i}\left[\frac{(n-1) U}{n}\right]^{i} E\left(X^{-i}\right)\right\} .
$$


Since

$$
E(\bar{X})^{-1}=\int_{0}^{\infty} \frac{1}{\Gamma(n)(\lambda / n)^{n}} \bar{X}^{(n-i)-1} e^{\frac{-\bar{X}}{(\lambda / n)}} d \bar{X}=\frac{\Gamma(n-i)}{\Gamma(n)(\lambda / n)^{i}},
$$

\section{Health examination for freshmen}

then $r$ th moment of $\tilde{S}_{I}$ can be obtained as follows:

$$
E\left(\tilde{S}_{1}\right)^{r}=\sum_{i=0}^{r} C_{i}^{r}\left\{(-1)^{r-i}\left[(n-1)\left(S_{I}+1\right)\right] \frac{{ }^{i}(n-i)}{\Gamma(n)}\right\} .
$$

859

\section{Appendix 4. $W$ conforms to Gamma $(n, 1)$}

Because $\bar{X}$ conforms to Gamma $(n, \lambda / n)$, let $W=n \bar{X} / \lambda$, and then the distribution of $W$ can be derived through transformations of random variables as follows:

$$
f_{W}(w)=f_{\bar{X}}(\bar{x})\left|\frac{d \bar{x}}{d w}\right|=f_{\bar{X}}\left(\frac{\lambda w}{n}\right) \cdot \frac{\lambda}{n}=\frac{1}{\Gamma(n)(\lambda / n)^{n}}(\lambda w / n)^{n-1} e^{-w} \cdot \frac{\lambda}{n}=\frac{1}{\Gamma(n)} w^{n-1} e^{-w} .
$$

Clearly, $W$ is conforming to $\operatorname{Gamma}(n, 1)$.

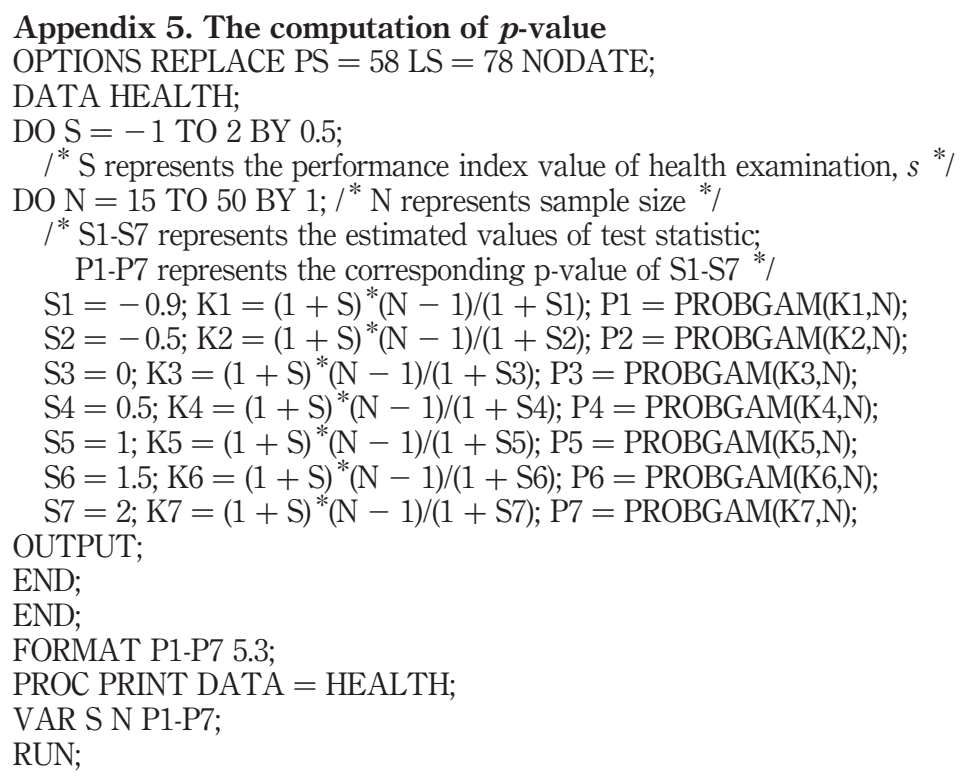

